Perspective

\title{
Continuous- versus Segmented-Flow Microfluidic Synthesis in Materials Science
}

\author{
Mathieu Gonidec ${ }^{1,2, *}$ and Josep Puigmartí-Luis ${ }^{3, *}$ (i) \\ 1 Centre National de la Recherche Scientifique (CNRS), Institut de Chimie de la Matière Condensée de \\ Bordeaux (ICMCB), UMR 5026, F-33600 Pessac, France \\ 2 University of Bordeaux, Institut de Chimie de la Matière Condensée de Bordeaux (ICMCB), UMR 5026, \\ F-33600 Pessac, France \\ 3 Institute for Chemical \& Bioengineering, Department of Chemistry \& Applied Biosciences, ETH Zurich, \\ 8093 Zurich, Switzerland \\ * Correspondence: mathieu.gonidec@icmcb.cnrs.fr (M.G.); josep.puigmarti@chem.ethz.ch (J.P.-L.); \\ Tel.: +41-44-632-30-64 (J.P.-L.)
}

Received: 12 November 2018; Accepted: 21 December 2018; Published: 24 December 2018

\begin{abstract}
Materials science is a fast-evolving area that aims to uncover functional materials with ever more sophisticated properties and functions. For this to happen, new methodologies for materials synthesis, optimization, and preparation are desired. In this context, microfluidic technologies have emerged as a key enabling tool for a low-cost and fast prototyping of materials. Their ability to screen multiple reaction conditions rapidly with a small amount of reagent, together with their unique physico-chemical characteristics, have made microfluidic devices a cornerstone technology in this research field. Among the different microfluidic approaches to materials synthesis, the main contenders can be classified in two categories: continuous-flow and segmented-flow microfluidic devices. These two families of devices present very distinct characteristics, but they are often pooled together in general discussions about the field with seemingly little awareness of the major divide between them. In this perspective, we outline the parallel evolution of those two sub-fields by highlighting the key differences between both approaches, via a discussion of their main achievements. We show how continuous-flow microfluidic approaches, mimicking nature, provide very finely-tuned chemical gradients that yield highly-controlled reaction-diffusion (RD) areas, while segmented-flow microfluidic systems provide, on the contrary, very fast homogenization methods, and therefore well-defined super-saturation regimes inside arrays of micro-droplets that can be manipulated and controlled at the milliseconds scale. Those two classes of microfluidic reactors thus provide unique and complementary advantages over classical batch synthesis, with a drive towards the rational synthesis of out-of-equilibrium states for the former, and the preparation of high-quality and complex nanoparticles with narrow size distributions for the latter.
\end{abstract}

Keywords: continuous-flow microfluidics; reaction-diffusion; segmented-flow microfluidics; controlled mixing; crystallization; self-assembly; kinetic control; out-of-equilibrium; pathway selection; pathway complexity

\section{Introduction}

In the last two decades, there has been a growing interest in using microfluidic devices for the synthesis of materials, the main reason being that microfluidic conditions offer significant advantages over macroscopic laboratory settings: reduced reactant consumption, high surface area to volume ratios, and improved control over mass and heat transfer are some of their unique assets [1,2]. In general, microfluidic devices handle reactions at the micro-scale, where some fluidic physical phenomena are 
dramatically different from those observed in macroscopic experiments [3-6]. For example, inside microfluidic devices, small volumes of reagent can be finely controlled and manipulated, enabling well-defined and highly reproducible reaction times and mixing conditions-features that have evoked a significant interest in materials synthesis via microfluidics means $[7,8]$.

Among all of the different microfluidic approaches employed for materials synthesis [9], herein we will focus our attention on continuous- and segmented-flow microfluidic devices used in the synthesis of crystalline materials. While those two configurations may not seem radically different at first glance-for instance, both situations correspond to very low Reynolds (Re) numbers and highly laminar flow conditions-they rely on fundamentally different processes for combining the reagents. Due to this apparent similarity, it is not instantly clear how radically different those approaches are, and this can lead to some confusion when discussing the outcome of such processes. Therefore, in this perspective, we aim to provide the reader with a unique viewpoint on the major differences between these two microfluidic configurations, by supporting our discussion with original data reported in the literature.

Laminar flow conditions present in continuous-flow microfluidic devices allow for a spatial and temporal control of reactants concentrations only through molecular diffusion (i.e., a controlled reaction-diffusion (RD) area is accomplished inside the microfluidic channel), while in segmented-flows (i.e., moving droplets or slugs), mixing is obtained by chaotic advection to achieve rapid mixing (Table 1 and Figure 1) [9,14]. That is why segmented-flow processes generated inside microfluidic devices-for which there is no controlled diffusion of reagents, and thus no control over the concentration gradients-have been used as screening platforms in crystallization process (e.g., protein crystallization). The fast and efficient homogenization of the reaction medium in these devices has, for example, facilitated the high throughput synthesis of materials with controlled morphology and size $[15,16]$, and the isolation and screening of multiple compartmentalized reactions in microdroplets $[17,18]$. In sharp contrast, continuous-flow microfluidic devices are experiencing relentless growth, because kinetic versus thermodynamic control in the formation of reaction products can be accomplished; a result that can potentially lead to new unprecedented materials and functions [19].

Table 1. Major differences/advantages for continuous- and segmented-flow microfluidic devices used for materials synthesis.

\begin{tabular}{|c|c|c|}
\hline $\begin{array}{c}\text { Type of } \\
\text { Microfluidic Device }\end{array}$ & Distinctive Features & $\begin{array}{c}\text { Materials } \\
\text { Synthetized/Morphology }\end{array}$ \\
\hline Continuous-flow & $\begin{array}{l}\text {-Mixing is slow and achieved only through } \\
\text { molecular diffusion. } \\
\text { - This condition allows to establish controlled } \\
\text { reaction-diffusion (RD) environments. } \\
\text { - The diffusion of reagents is controlled in space } \\
\text { and time avoiding chaotic advection. } \\
\text { - A parabolic profile of fluid velocity vectors } \\
\text { inside the microfluidic channel. } \\
\text {-It is difficult to upscale the synthesis } \\
\text { of materials. }\end{array}$ & $\begin{array}{l}\text { Organic, inorganic, and composite } \\
\text { materials / Continuous fibers } \\
{[10,11] \text { and continuous }} \\
\text { membranes }[12,13] \text { can } \\
\text { be generated. }\end{array}$ \\
\hline Segmented-flow & $\begin{array}{l}\text { - Reagents can be efficiently mixed on } \\
\text { sub-millisecond time scales via chaotic advection, } \\
\text { and mixing can be programmed. } \\
\text { - The no-slip boundary condition is omitted. } \\
\text { —Consecutive reactions can be synchronized to } \\
\text { synthetize core-shell particles and crystals. } \\
\text {-Multiple reactions can be screened rapidly and } \\
\text { while employing small amounts of reagents. }\end{array}$ & $\begin{array}{l}\text { Organic, inorganic, and composite } \\
\text { materials/This approach does not } \\
\text { allow the formation of continuous } \\
\text { fibers or continuous membranes. }\end{array}$ \\
\hline
\end{tabular}

An important goal in material science, and hence for nanotechnology development, is to control not only the size and shape of the functional materials produced, but their physico-chemical properties. 
Conventionally, materials' properties are tuned via chemical variations of molecular building blocks, or by changing reaction conditions (such as temperature, pressure, solvent composition, among others).

(a)

Segmented
Flow
Continuous
Flow
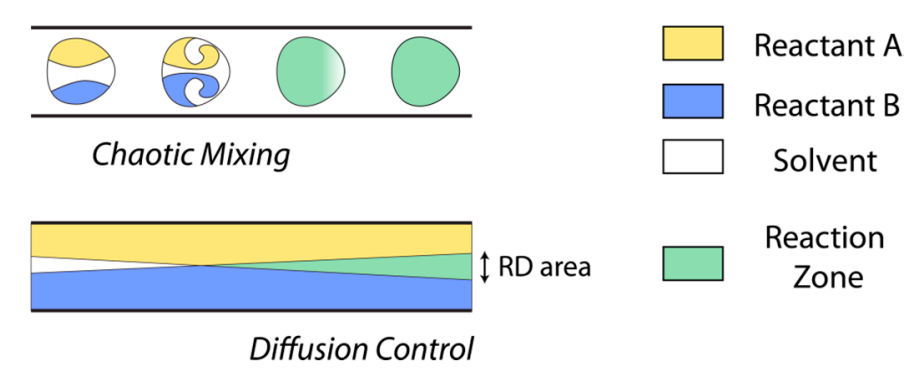

Figure 1. Comparison of reactants mixing in segmented- and continuous-flow microfluidic devices. As shown in this figure, in segmented-flow devices (a), mixing is due to chaotic advection, whereas in continuous-flow microfluidic devices (b), mixing of reactants is only accomplished through molecular diffusion. Note that the latter is a unique method for establishing an effective reaction-diffusion (RD) area, just like the ones present in nature. In the figure, the yellow and blue colors indicate reactants, the white color the pure solvent area, and in green is the reaction area.

Therefore, it is highly desirable to provide novel tools that can define and guarantee a reproducible formation pathway for a wide library of experimental conditions, as well as technologies enabling the isolation of materials far away from the global minimum of Gibbs energy (i.e., to achieve out-of-equilibrium species). Here, we will show that continuous and segmented-flow microfluidic devices have emerged as landmark technologies for both (i) the capture of kinetic and metastable states (continuous-flow), and (ii) the discovery and production of materials with controlled sizes and shapes (segmented-flow), thus opening new vistas to the materialization of novel structure-property correlations in existing artificial compounds.

\section{Continuous Flow}

The turbulence-free condition governing continuous-flow microfluidic devices-where mixing is only driven by molecular diffusion (Table 1 and Figure 1 b) — has evoked multiple studies for materials synthesis. Since the micro-fabrication of silver "wires" and inorganic microcrystal arrays (e.g., calcite and apatite) in 1999 by the Whitesides group [20], many research laboratories have investigated the formation and assembly of a wide variety of materials, ranging from single molecular building blocks, such as lipids [7], to large macromolecules (e.g., polymers [21]) in two laminar co-flowing streams or in flow-focusing microfluidic set-ups (Figure 2a,b, respectively).

As we will show in this section, the laminar flow condition found in continuous-flow microfluidic devices enables the controlled formation of what we call tunable and self-engineered reaction-diffusion (RD) environments. RD conditions, generated inside microfluidic channels, mimic processes found in nature that are key to accomplish changes in crystal structures, and hence, in specific functions of composite materials. For example, seashells (a composite material) exhibit high toughness because calcite crystals and aragonite crystals are generated and oriented out-of-plane and in-plane, respectively, via RD environments [22]. In this vein, in 2010, Puigmartí et al. demonstrated that microfluidic platforms operating under RD conditions can advantageously be used to generate different crystal packings and morphologies of the same material, just by varying the flow rate ratio (FRR) during synthesis [23]. The FRR is defined as the ratio between the sheath and reagent flows. In this work, the authors employed a planar four-inlet microfluidic device, where solutions of tetrathiafulvalene (TTF) and a gold (Au) salt in acetonitrile were injected in the middle channels, while acetonitrile sheath flows were used to focus or widen the RD area in the main microfluidic channel, i.e., where the reaction materialized (Figure 2b). Varying the FRR between 0.1 and 14, significant changes in the shape of the resulting TTF-Au composite crystals were observed (Figure 2c). While at a low FRR non-uniform 
crystal habits were obtained, after increasing the FRR short wires with large diameters and rod-like structures were synthetized (Figure 2c). Additionally, at an FRR of 14, thin and long nanowires were fabricated (ca. $200 \mathrm{~nm}$ in width and tens of micrometers in length). Note that these structures could not be generated in conventional synthetic approaches, such as turbulent bulk mixing experiments.

(a)

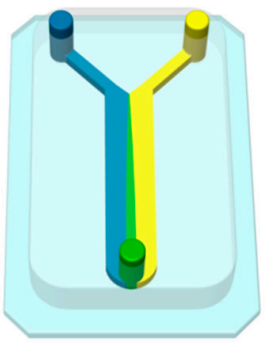

(b)

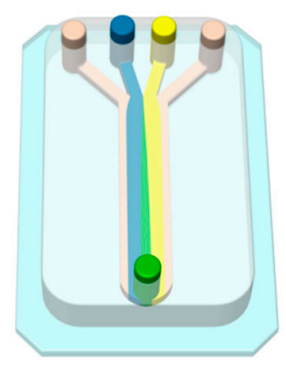

(c)

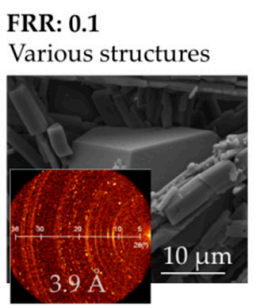

FRR: 0.5 - 2 Rods / hollow tubes

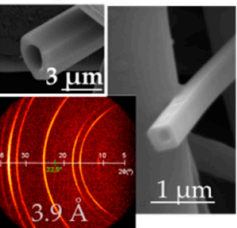

(d)

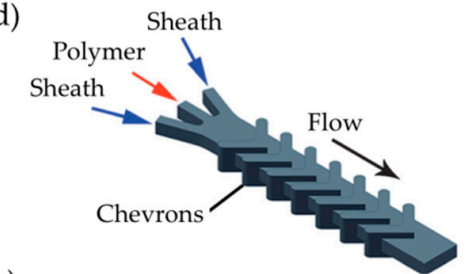

(e)

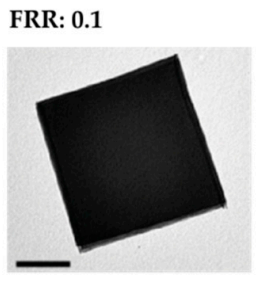

FRR: 1

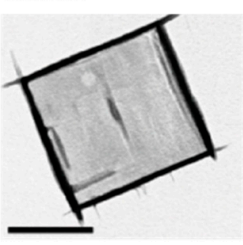

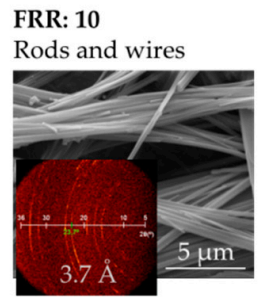

FRR: 14

Mainly long wires
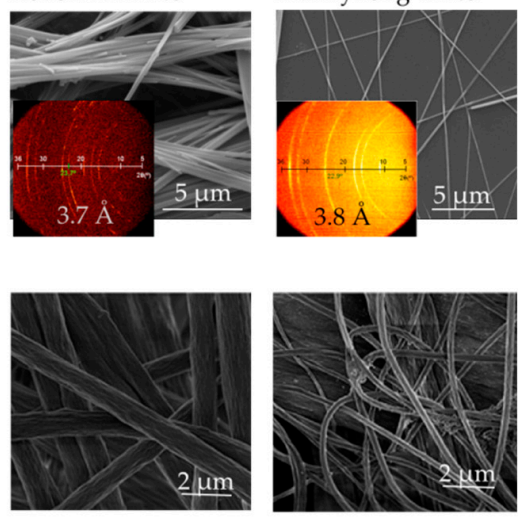

FRR: 4

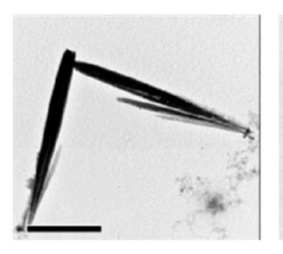

FRR: 5

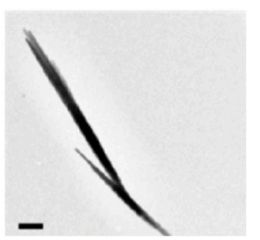

Figure 2. Schematic illustrations of (a) two laminar co-flowing streams and (b) a flow-focusing microfluidic set-up (or hydrodynamic focusing). As in Figure 1, the yellow and blue colors indicate reactants, white is the pure solvent, and green the reaction area where RD conditions are settled. In (c) are scanning electron microscopy (SEM) images of TTF-Au composites generated at flow rate ratios (FRRs) of $0.1,0.5-2,10$ and 14, with their respective two-dimensional (2D) X-ray diffraction (XRD) spectra. Each 2D XRD spectra indicates the pi-stacking distance between TTF molecules in the composite (reproduced with permission from Wiley-VCH [23]). (d) Drawing of a flow-focusing device incorporating chevrons and SEM images of the poly(methylmethacrylate) PMMA fibers synthetized (Reproduced with permission from RSC [10]). (e) Transmission electron microscopy (TEM) images of the coordination polymers (CP) synthetized at different FRRs. Here, the scale bars are $1 \mu \mathrm{m}$. (Reproduced with permission from Wiley- $\mathrm{VCH}[24]$ ).

Surprisingly, this work also proved that $\mathrm{RD}$ conditions can be used as an advanced micro-engineering tool to guide self-assembly. Indeed, it was demonstrated that different FRRs led to changes in the crystal structure of the final TTF-Au composites. Herein, the pi-stacking distance between TTF molecules in the final composite material was modified. These variations in the crystal structure were only the end result of changes in the flowing conditions during synthesis. Accordingly, the authors did not have to change the functional groups present in the TTF molecules, nor the synthetic conditions (solvent, temperature, or pressure) to accomplish different crystal structures and morphologies in the final TTF-Au composite products.

Control over the size and shape of macromolecules (e.g., polymers) has also been a matter of intense investigation with continuous-flow microfluidic devices. For example, Ligler and co-workers demonstrated that the cross-sectional shape of fibers fabricated from a model polymer (e.g., poly(methylmethacrylate) (PMMA)) can be accurately defined by changing the FRR between sheath flows and the polymer-laden flow during synthesis (Figure 2d) [10]. In their work, the authors 
made use of hydrodynamic flow-focusing devices, incorporating grooves into the channel walls that facilitated a controlled deflection of the different fluid streams along the main microfluidic channel. They observed that this microfluidic sheathing device integrating grooves can produce PMMA fibers of ca. $300 \mathrm{~nm}$ in width or ribbon-shaped PMMA fibers (Figure 2d). The dimensions and shape of the PMMA fibers described were accurately controlled by the number of grooves and their configuration (chevron pattern or diagonal) inside the microfluidic channel where the solidification (or polymerization) occurs.

Recently, continuous-flow microfluidic devices have also been employed for the synthesis of a new type of covalent organic polymer, also known as covalent organic frameworks (COFs). COFs are crystalline porous materials that are frequently synthetized under solvothermal conditions, yielding insoluble and un-processable powders; features that have hindered their widespread application. In this context, Puigmartí et al. demonstrated that continuous-flow microfluidic devices can circumvent these limitations associated with COF synthesis. [11] In their seminal work, the authors showed that continuous-flow microfluidic synthesis enables the generation of COF fibers at room temperature, and that these can be directly printed in 2D and three-dimensional (3D) structures on a surface. Surprisingly, they also observed that the COFs synthetized with the microfluidic approach were formed of interconnected nanoscale fibers that resulted into a 3D microporous sponge-like material, and that this morphology was completely different from the one obtained under conventional synthetic methods, where only a powder with a smooth morphology was characterized. Even though RD conditions are still in their infancy for materials synthesis, their universality was demonstrated with the synthesis of other functional materials like coordination polymers $(\mathrm{CP})$, with the first contribution dating back to 2011 [25]. In this case, the authors described the use of continuous-flow microfluidic devices (with a flow focusing configuration (Figure 2b) to fabricate 1D nanoscaled CPs with an unprecedented morphology and under exceptional controlled conditions (i.e., parallelized synthesis, controlled contact time of two reagent streams, controlled diffusion of species, and space localization of the coordination pathway). For example, they showed that while the bulk synthesis of a CP made of $\mathrm{Ag}(\mathrm{I})$ ions and the amino acid cysteine (Cys) led to membrane-like structures, the microfluidic synthesis in controlled RD conditions produced long Ag-(I)-Cys nanofibers. Moreover, this effect was also extended to other CPs, hence showing the generality of this approach to favoring the supramolecular assembly of long one-dimensional (1D) nanoscale structures under microfluidic RD environments. These results represent a step forward for the construction of novel metal-organic supramolecular nano-assemblies based on coordination chemistry, which could unveil new properties in these materials.

In 2016, the same group showed that not only 1D nanoscale CP structures are generated under RD conditions, but out-of-equilibrium CP crystal structures can also be accomplished [24]. In this study, the authors show that the microfluidic synthesis (under RD conditions) of a CP constructed from $\mathrm{Cu}(\mathrm{II})$ ions and 4,4'-bipyridine linkers leads to different crystal habits. For example, they observed that needles are generated at a high FRR (i.e., at an FRR of 5); partial square crystals at an FRR of 4; square partially-filled crystals at anFRR of 1 ; and finally, completely filled square plate-like crystals are synthesized at an FRR of 0.1 (Figure 2e). Importantly, the authors highlight the fact that only filled square plate-like crystals are obtained by conventional diffusion or by flask mixing experiments in macroscopic settings. Accordingly, it was not possible to isolate any of the intermediate crystals shown in Figure 2e employing known established methods and protocols, such as, for example, the addition of surfactants during the crystallization process. In a crystallization process, the faces with a higher energy are the ones determining the crystal growth, morphology, aspect ratio, and the final crystallographic order. To modify the relative energies of crystal faces is difficult under conventional synthetic methods-only the addition of chemical impurities like surfactant molecules and polymers has been shown to be efficient [26]. Additionally, the authors demonstrated in that study that all the structures generated with the continuous-flow microfluidic device have the same diffraction pattern, and that it is identical to the one measured for bulk samples. 
Last but not least, we would like to highlight in this section that continuous-flow microfluidic devices have also found application in the fabrication of molecular-based sieving membranes (Table 1). Molecular sieving membranes have experienced considerable interest in both industry and academia, as they can be efficiently used as separation systems in chemistry and petro-chemistry [27]. However, the fabrication costs of these membranes are high, and their production rate is low. In this vein, Nair et al. showed that a continuous-flow microfluidic device can overcome these barriers by synthetizing a chemically stable, thermally resistant, and high-performance separation membrane based on a prototypal metal organic framework (MOF), specifically zeolitic imidazolate framework (ZIF)-8 [12]. MOFs are a type of porous material constructed from organic ligands linked to metal ions (or clusters), which that have found application as energy-efficient and environmentally friendly gas separation matter [28]. Therefore, the implementation of MOFs in membrane-like structures has increased during the last decade. In their work, the authors demonstrated that the high control over mass transport and reactant availability during the formation of MOF crystals enabled the fabrication of a highly efficient molecular sieving MOF membrane, at low cost and with a high potential for parallelization (i.e., scale-up production). Note that in their approach, the authors achieved a controlled growth of a ZIF-8 layer inside a porous polymeric and hollow fiber by flowing the metal ion solution inside the fiber while the organic ligand was supplied by dipping the fiber in a conventional beaker. Recently, this approach has also been extended. For example, Coronas and coworkers have demonstrated that the controlled growth of MOF layers inside a hollow polymeric fiber can be accomplished by pumping both reagents inside the fiber [13]. This method not only reduces the reactant consumption, but can ensure a better control over the MOF growth, hence facilitating thinner and more highly-efficient MOF membranes.

\section{Segmented-Flow Microfluidics}

In a seminal paper in 2001, Thorsen et al. paved the way for modern droplet microfluidic by demonstrating that it was possible to produce highly stable and ordered streams of droplets in a T-junction generator [29]. This result spawned a flurry of research efforts focusing on the use of droplets as well-defined micro-environments in which to carry out reactions [30-35] or crystallizations [17,18,36-38]. Essentially, many began to see droplets as miniaturized units replacing conventional batch chemistry glassware, for which the figures of merit (such as mixing efficacy and the timescale at which one could perform unit operations) were the same as the ones used thus far, but with the hope of achieving greater control over the process parameters. Indeed, in segmented flow devices, reagents or compounds of interest can be mixed in minute amounts and compartmentalized. When studying synthesis or crystallization conditions, this means that one could, in principle, screen a great number of reaction conditions, using either individual droplets or batches of droplets for each set of parameters. Moreover, unlike batch chemical approaches, once adequate conditions are found, one can easily scale up the process (by either parallelization of devices [39] or by simply extending the operation time) without affecting the reaction conditions, and the high degree of control achieved at low dimensions would ensure the reproducibility of the process. With those strategies in mind, the main aspects to consider are (i) the efficiency of the mixing of the reagent streams, and (ii) the possibility to manipulate the droplet at very high temporal resolution to perform multiple on-chip unit operations.

In microfluidic systems, due to the very low Re numbers characterizing the flows, mixing cannot rely on turbulent convection. To produce homogeneous reaction mixtures, an efficient mixing process is therefore required, and studying mixing and finding conditions in which the process is fast and efficient was a key focus of microfluidic research in the early 2000s. This can be achieved in continuous-flow devices by using sophisticated designs, such as striated channels acting as chaotic mixers [43] or complex 3D chips consisting of a series of split and recombine units that essentially fold the laminated flow repeatedly [44-46]. In droplet microfluidics, due to the biphasic nature of the flow, mixing occurs spontaneously, even in simple, straight channels (see Figure 3a) [14,40], and modelling has shown that 
complete mixing is typically observed for a travel length to channel width ratio of 20. For example, and as shown in Figure 3b, a droplet needs to travel ca. $1 \mathrm{~mm}$ inside a $50 \mu \mathrm{m}$ channel to efficiently mix. Additionally, one can increase the mixing in a droplet by designing zig-zag channels with sharp turns that provide enhanced chaotic mixing by alternatively folding the flow in one direction and the other (see Figure 3c,d) [41,42,47-49]. The mixing efficiency depends critically on many parameters, including the capillary number $(\mathrm{Ca})$, the ratio of viscosities of the dispersed and the continuous phases, and the ratio of the drop diameter and the channel width [47]. A few detailed theoretical analyses have allowed to rationalize the streamlines generated inside microdroplets, [50,51], and to study the influence of different flow parameters on the mixing efficiency in corrugated channels [47,52]. More specifically, mixing is more efficient at very low capillary numbers, is optimal for droplet diameters about half as big as the winding channel widths, and is significantly more efficient when the viscosity ratio between the two fluids is low (i.e., when the viscosity of the dispersed phase is significantly lower than that of the continuous phase).

(a)
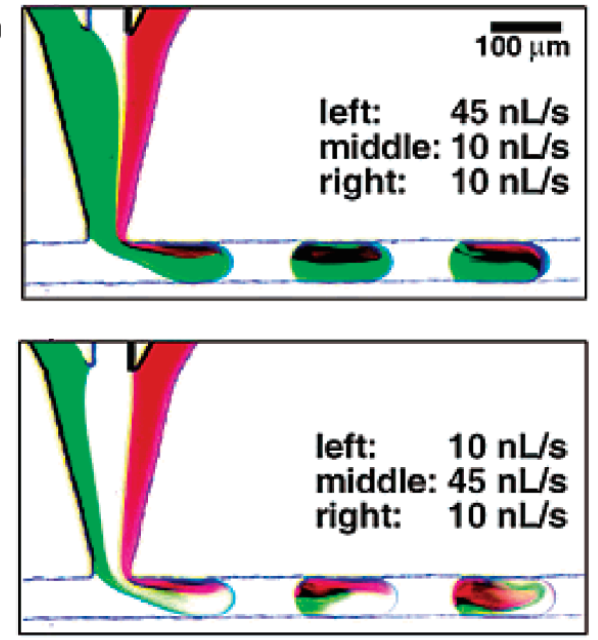

(c)
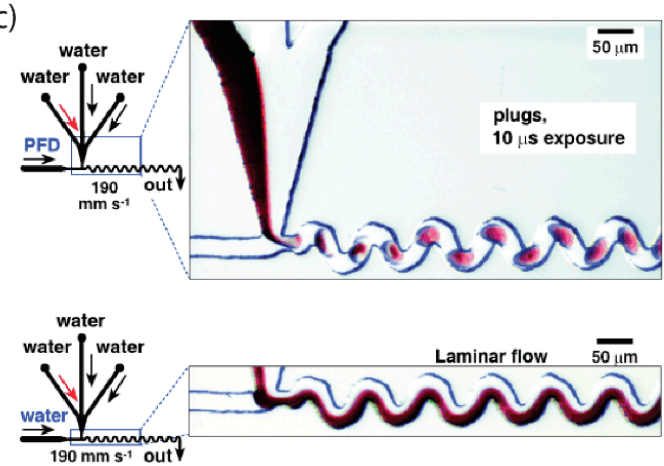

(b)

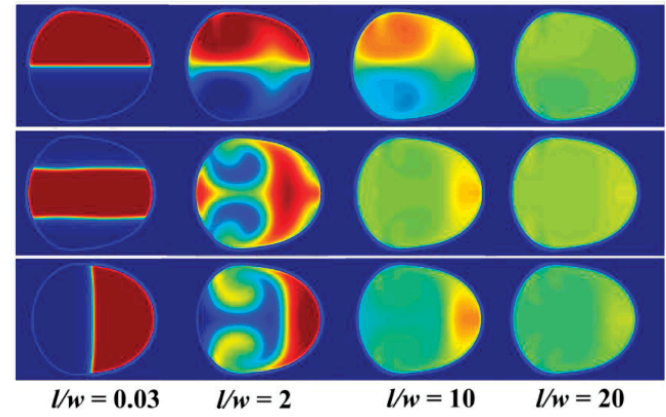

(d)

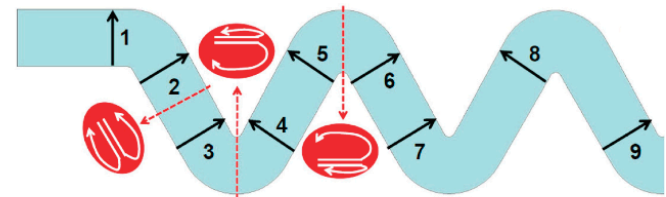

(e)

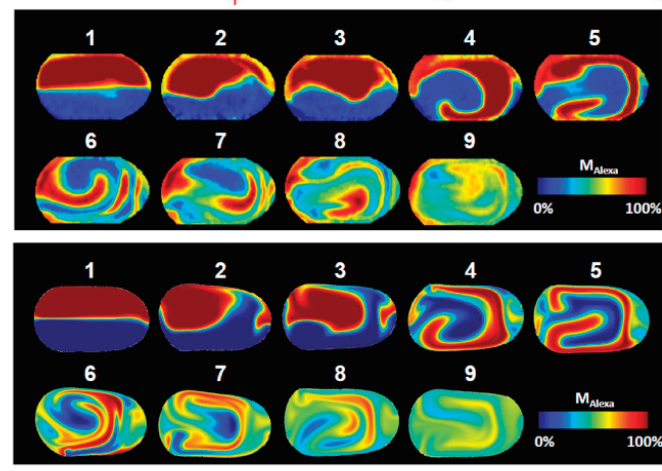

Figure 3. Spontaneous mixing of droplets flowing in straight channels for different (a) experimental (reprinted with permission from [14], copyright 2003 American Chemical Society), and (b) theoretical studies (reprinted from [40] with permission from Elsevier). While some mixing occurs reasonably fast (i.e., in a few ms) in such conditions, forced chaotic mixing in winding channels has been observed experimentally (c) (Copyright Wiley-VCH Verlag GmbH and Co., KGaA; reproduced with permission from [41]) and the mixing dynamics have been confirmed and analyzed in depth by theoretical calculations (d) (reprinted from [42], with the permission of AIP Publishing). (e) sequence of micrographs of the finite element analysis of the mixing occurring inside a droplet in the winding channel shown in (d).

The possibility of obtaining very fast and reproducible homogenization of the reaction medium has many important implications. For instance, it has allowed many groups to prepare high-quality 
nanoparticles of many types [30,53]—including MOFs, quantum dots, metallic nanoparticles, porous zeolites [54], and oxides-with a good rational control over particle sizes [55], as well as with narrower size dispersion with respect to similar synthesis performed with batch methods and therefore better defined properties $[31,39]$. The possibility to work on a very small scale, combined with the ease of in situ adjustment of the critical experimental parameters (such as the concentration of reagents, the flow rates, and the residence time), have opened the way to an easy screening of the reaction conditions, and therefore to improved capabilities in tuning the particle sizes (see Figure 4a) [55].

(a)

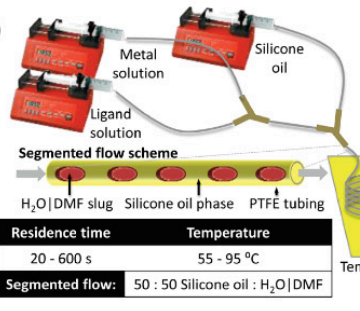

(c)
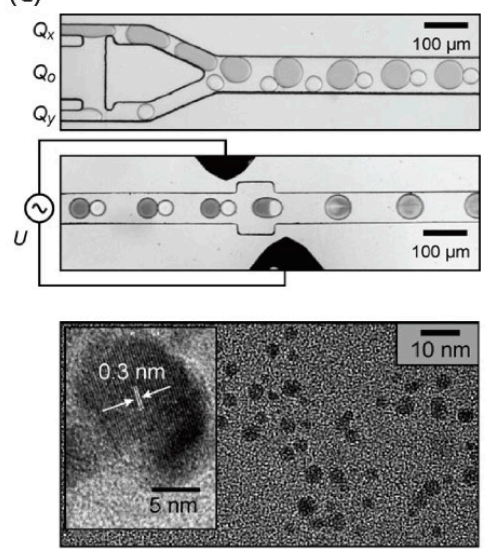

(b)
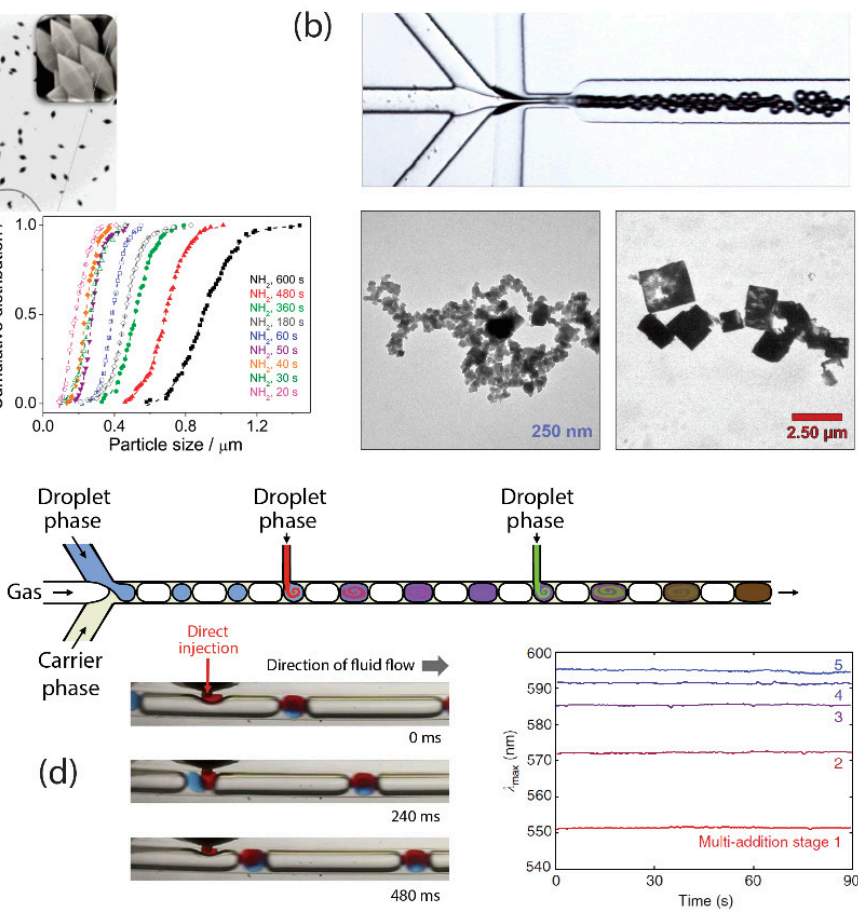
phase

Figure 4. (a) Rational control of metal organic framework (MOF) crystal sizes by droplet microfluidics (adapted with permission from [55], copyright 2013 American Chemical Society). (b) Chip design and comparative TEM images of the particles of a spin crossover coordination polymer, obtained by microfluidic (left) and batch (right) syntheses (adapted from [35]). The microfluidic approach yield nanoparticles with 20-fold downsizing with respect to the batch reaction. (c) Droplet pair generator (Copyright Wiley-VCH Verlag $\mathrm{GmbH}$ and Co. $\mathrm{KGaA}$; reproduced with permission from [56]) and its application to synthetize monocrystalline iron oxide nanoparticles (copyright Wiley-VCH Verlag $\mathrm{GmbH}$ and Co. KGaA; reproduced with permission from [32]). (d) Rational, sequential synthesis of cadmium selenide (CdSe) quantum dots by successive injections of reagents inside droplets (adapted from [57]).

In most cases, there is no major difference in particle size between microfluidic and batch methods, and the major improvement lies in the control of the size dispersion. However, González et al. [35] recently reported a microfluidic synthesis of spin-crossover coordination polymer nanoparticles, using a flow-focusing droplet nozzle, for which the opposite effect was observed. The size dispersion was essentially similar to that of the batch synthesis conditions, but the authors observed a 20-fold reduction in the size of the nanocrystals with respect to the same reaction carried out in simple reaction flasks (see Figure $4 b$ ). The reasons for this effect are yet speculative, but they are likely due to either enhanced mixing conditions, which could prove critical for the compound they produced, due to very fast reaction kinetics involved, or to complex effects linked to the rate of heterogeneous nucleation inside micro-droplets. Disentangling those different contributions will likely require a more extensive analysis of the influence of droplet sizes (that can affect heterogeneous nucleation rates) and the use of different corrugated channels (to modulate the mixing efficiencies). 
Beyond mixing aspects in droplets formed directly from a co-flowing stream of multiple reagents, droplet microfluidics also provide a second unique feature that has attracted the interest of the materials-science community: the possibility to manipulate droplets (i.e., to split or combine them in a highly controlled manner, and to inject reagents into pre-formed droplets). For instance, Frenz et al. demonstrated that one can prepare perfectly synchronized droplet pairs with a dual nozzle [56], and that such droplet pairs could then be fused at will (by destabilizing them with an applied electric field) to mix the reagents they contain. Using this concept (see Figure 4c), and thanks to the highly efficient mixing occurring during droplet fusion, the authors were able to synthesize high-quality monocrystalline iron oxide nanoparticles [32].

Another degree of complexity can be achieved by performing multiple operations-including multi-step reactions and quenching steps-at the milliseconds timescale, in a single chip, as demonstrated by the group of Ismagilov [14,31,41]. In particular, the group showed that instead of preparing separate droplets of reagents and fusing them, one can instead choose to inject a reagent directly into each individual droplet (or slugs) in a stream, and that such a system self-synchronizes [58]. Such methods have been used by many authors to perform multi-step reactions, including quenching steps to stop the reactions at the desired stage of growth [31], and to prepare core-shell particles of a variety of materials including quantum dots [31] and MOFs [34]. However, maybe the most impressive result with direct injection is a recent work from the deMello group, which used a three-phase droplet scheme (using air plugs to separate droplets of reagents) to perform multiple additions inside of streams of droplet microfluidic devices, achieving a good control over successive growth steps in the continuous preparation of CdSe quantum dots, and thus ensuring great control over their luminescence properties (see Figure 4d) [57]. This strategy, and the high-quality growth conditions it exhibits, is a very powerful tool, and it could be extended to the rational engineering of core-shell nanoparticles.

\section{Conclusions and Perspectives}

Altogether, over the last two decades, continuous-flow and segmented-flow microfluidic approaches have provided many interesting leads for the synthesis of functional crystalline materials, thanks to either the extreme control in RD zones yielded by continuous-flow methods, or to the high convective mixing efficiencies afforded by droplet-based approaches.

Even though continuous-flow microfluidic devices have proven to be an efficient method to mimic RD environments present in nature, a major challenge will be to use these bio-inspired conditions to fine-tune the properties of man-made materials just like nature does (e.g., in biomineralization processes). Indeed, this objective will be accomplished when scientist will have full control over the energy landscape of a given system. Accordingly, one could decide the pathway that the system should follow (pathway selection) to obtain a specific state (thermodynamic, kinetic, or metastable) with a particular property. Recently, most artificial materials produced in laboratories around the world are obtained at thermodynamic equilibrium, where materials' properties and functions are fixed. Therefore, there is a great interest in the scientific community to find new and robust ways to go out of equilibrium during self-assembly processes. The main reason for this is that out-of-equilibrium states will not only open new routes to new materials with novel functions, but they will be key to understanding and unveiling pathway complexity in artificial systems.

Droplet-based synthetic methods have relied heavily on empirical findings, and have paved the way for a high-quality rational design of particle sizes and shapes, with improved quality factors with respect to batch methods. Nevertheless, there are still major hurdles to overcome in the understanding of reactivity and crystallization at the microscale, in order to be able to perform rational syntheses of new compounds without long empirical adjustments of chip geometries and operating conditions. In particular, for reactions evidencing fast kinetics of the order of the mixing time (i.e., a few ms), it is still unclear if the size of the particles can be ultimately controlled by the mixing process itself, or by the control of the heterogeneous and homogeneous nucleation rates. Beyond those fundamental aspects, engineering crystalline nanoparticles could, in principle, be done by performing reactions in 
ultra-small droplets (in the micrometer or sub-micrometer range), which poses significant experimental challenges. Improvement in all of those directions, combined with an exquisite understanding and control of nucleation and growth conditions, will likely result, in the near future, in the ability to synthesize core-shell nanocrystals with arbitrary composition, sizes, and dimensions.

Author Contributions: J.P.-L. and M.G. contributed equally to the writing of the paper. J.P.-L. wrote the "Continuous Flow" section and M.G. the "Segmented-Flow Microfluidics" part.

Funding: J.P.L. acknowledges funding from the European Research Council (ERC-2015-STG No. 677020), the Swiss National Science Foundation (project no. 200021_160174) and networking support by the intergovernmental framework for European Cooperation in Science and Technology Action (COST) CM1402 "Crystallize". M.G. acknowledges funding from the French National Research Agency (ANR) Investment for the Future Programme IdEx Bordeaux (ANR-10-IDEX-03-02).

Conflicts of Interest: The authors declare no conflict of interest.

\section{References}

1. Ehrfeld, W.; Hessel, V.; Löwe, H. Microreactors: New Technology for Modern Chemistry; Wiley-VCH: New York, NY, USA, 2000; ISBN 9783527295906.

2. Jensen, K. Chemical kinetics: Smaller, faster chemistry. Nature 1998, 393, 735-737. [CrossRef]

3. Squires, T.M.; Quake, S.R. Microfluidics: Fluid physics at the nanoliter scale. Rev. Mod. Phys. 2005, 77, 977-1026. [CrossRef]

4. Nguyen, N.; Wereley, S. Fundamentals and Applications of Microfluidics; Artech House: Boston, MA, USA, 2006; ISBN 1580539726.

5. Purcell, E.M. Life at low Reynolds number. Am. J. Phys. 1977, 45, 3-11. [CrossRef]

6. Elvira, K.S.; I. Solvas, X.C.; Wootton, R.C.R.R.; DeMello, A.J. The past, present and potential for microfluidic reactor technology in chemical synthesis. Nat. Chem. 2013, 5, 905-915. [CrossRef] [PubMed]

7. Jahn, A.; Vreeland, W.N.; Gaitan, M.; Locascio, L.E. Controlled Vesicle Self-Assembly in Microfluidic Channels with Hydrodynamic Focusing. J. Am. Chem. Soc. 2004, 126, 2674-2675. [CrossRef] [PubMed]

8. Dendukuri, D.; Pregibon, D.C.; Collins, J.; Hatton, T.A.; Doyle, P.S. Continuous-flow lithography for high-throughput microparticle synthesis. Nat. Mater. 2006, 5, 365-369. [CrossRef]

9. Puigmartí-Luis, J. Microfluidic platforms: A mainstream technology for the preparation of crystals. Chem. Soc. Rev. 2014, 43, 2253-2271. [CrossRef]

10. Thangawng, A.L.; Howell, P.B., Jr.; Richards, J.J.; Erickson, J.S.; Ligler, F.S. A simple sheath-flow microfluidic device for micro/nanomanufacturing: Fabrication of hydrodynamically shaped polymer fibers. Lab Chip 2009, 9, 3126. [CrossRef]

11. Rodríguez-San-Miguel, D.; Abrishamkar, A.; Navarro, J.A.R.; Rodriguez-Trujillo, R.; Amabilino, D.B.; Mas-Ballesté, R.; Zamora, F.; Puigmartí-Luis, J. Crystalline fibres of a covalent organic framework through bottom-up microfluidic synthesis. Chem. Commun. 2016, 52, 9212-9215. [CrossRef]

12. Brown, A.J.; Brunelli, N.A.; Eum, K.; Rashidi, F.; Johnson, J.R.; Koros, W.J.; Jones, C.W.; Nair, S. Interfacial microfluidic processing of metal-organic framework hollow fiber membranes. Science 2014, 345, 72 LP-75. [CrossRef]

13. Cacho-Bailo, F.; Catalán-Aguirre, S.; Etxeberría-Benavides, M.; Karvan, O.; Sebastian, V.; Téllez, C.; Coronas, J. Metal-organic framework membranes on the inner-side of a polymeric hollow fiber by microfluidic synthesis. J. Memb. Sci. 2015, 476, 277-285. [CrossRef]

14. Song, H.; Ismagilov, R.F. Millisecond Kinetics on a Microfluidic Chip Using Nanoliters of Reagents. J. Am. Chem. Soc. 2003, 125, 14613-14619. [CrossRef] [PubMed]

15. Chan, E.M.; Alivisatos, A.P.; Mathies, R.A. High-Temperature Microfluidic Synthesis of CdSe Nanocrystals in Nanoliter Droplets. J. Am. Chem. Soc. 2005, 127, 13854-13861. [CrossRef] [PubMed]

16. Abou-Hassan, A.; Sandre, O.; Cabuil, V. Microfluidics in Inorganic Chemistry. Angew. Chem. Int. Ed. 2010, 49, 6268-6286. [CrossRef] [PubMed]

17. Zheng, B.; Roach, L.S.; Ismagilov, R.F. Screening of Protein Crystallization Conditions on a Microfluidic Chip Using Nanoliter-Size Droplets. J. Am. Chem. Soc. 2003, 125, 11170-11171. [CrossRef]

18. Lau, B.T.C.; Baitz, C.A.; Dong, X.P.; Hansen, C.L. A Complete Microfluidic Screening Platform for Rational Protein Crystallization. J. Am. Chem. Soc. 2007, 129, 454-455. [CrossRef] [PubMed] 
19. Sevim, S.; Sorrenti, A.; Franco, C.; Furukawa, S.; Pané, S.; de Mello, A.J.; Puigmartí-Luis, J. Self-assembled materials and supramolecular chemistry within microfluidic environments: From common thermodynamic states to non-equilibrium structures. Chem. Soc. Rev. 2018, 47, 3788-3803. [CrossRef]

20. Kenis, P.J.A.; Ismagilov, R.F.; Whitesides, G.M. Microfabrication inside capillaries using multiphase laminar flow patterning. Science 1999, 285, 83-85. [CrossRef]

21. Hou, X.; Zhang, Y.S.; Santiago, G.T.; Alvarez, M.M.; Ribas, J.; Jonas, S.J.; Weiss, P.S.; Andrews, A.M.; Aizenberg, J.; Khademhosseini, A. Interplay between materials and microfluidics. Nat. Rev. Mater. 2017, 2, 17016. [CrossRef]

22. Fleischli, F.D.; Dietiker, M.; Borgia, C.; Spolenak, R. The influence of internal length scales on mechanical properties in natural nanocomposites: A comparative study on inner layers of seashells. Acta Biomater. 2008, 4, 1694-1706. [CrossRef]

23. Puigmartí-Luis, J.; Schaffhauser, D.; Burg, B.R.; Dittrich, P.S. A Microfluidic Approach for the Formation of Conductive Nanowires and Hollow Hybrid Structures. Adv. Mater. 2010, 22, 2255-2259. [CrossRef] [PubMed]

24. Rubio-Martinez, M.; Imaz, I.; Domingo, N.; Abrishamkar, A.; Mayor, T.S.; Rossi, R.M.; Carbonell, C.; DeMello, A.J.; Amabilino, D.B.; Maspoch, D.; et al. Freezing the Nonclassical Crystal Growth of a Coordination Polymer Using Controlled Dynamic Gradients. Adv. Mater. 2016, 28, 8150-8155. [CrossRef] [PubMed]

25. Puigmartí-Luis, J.; Rubio-Martínez, M.; Hartfelder, U.; Imaz, I.; Maspoch, D.; Dittrich, P.S. Coordination Polymer Nanofibers Generated by Microfluidic Synthesis. J. Am. Chem. Soc. 2011, 133, 4216-4219. [CrossRef] [PubMed]

26. Jun, Y.; Casula, M.F.; Sim, J.-H.; Kim, S.Y.; Cheon, J.; Alivisatos, A.P. Surfactant-Assisted Elimination of a High Energy Facet as a Means of Controlling the Shapes of $\mathrm{TiO}_{2}$ Nanocrystals. J. Am. Chem. Soc. 2003, 125, 15981-15985. [CrossRef] [PubMed]

27. Bastani, D.; Esmaeili, N.; Asadollahi, M. Polymeric mixed matrix membranes containing zeolites as a filler for gas separation applications: A review. J. Ind. Eng. Chem. 2013, 19, 375-393. [CrossRef]

28. Li, J.-R.; Kuppler, R.J.; Zhou, H.-C. Selective gas adsorption and separation in metal-organic frameworks. Chem. Soc. Rev. 2009, 38, 1477. [CrossRef] [PubMed]

29. Thorsen, T.; Roberts, R.W.; Arnold, F.H.; Quake, S.R. Dynamic Pattern Formation in a Vesicle-Generating Microfluidic Device. Phys. Rev. Lett. 2001, 86, 4163-4166. [CrossRef] [PubMed]

30. Marre, S.; Jensen, K.F. Synthesis of micro and nanostructures in microfluidic systems. Chem. Soc. Rev. 2010, 39, 1183. [CrossRef]

31. Shestopalov, I.; Tice, J.D.; Ismagilov, R.F. Multi-step synthesis of nanoparticles performed on millisecond time scale in a microfluidic droplet-based system. Lab Chip 2004, 4, 316. [CrossRef]

32. Frenz, L.; El Harrak, A.; Pauly, M.; Bégin-Colin, S.; Griffiths, A.D.; Baret, J.C. Droplet-based microreactors for the synthesis of magnetic iron oxide nanoparticles. Angew. Chem. Int. Ed. 2008, 47, 6817-6820. [CrossRef]

33. Hung, L.-H.; Choi, K.M.; Tseng, W.-Y.; Tan, Y.-C.; Shea, K.J.; Lee, A.P. Alternating droplet generation and controlled dynamic droplet fusion in microfluidic device for CdS nanoparticle synthesis. Lab Chip 2006, 6, 174-178. [CrossRef] [PubMed]

34. Faustini, M.; Kim, J.; Jeong, G.; Kim, J.Y.; Moon, H.R.; Ahn, W.; Kim, D. Microfluidic Approach toward Continuous and Ultrafast Synthesis of Metal-Organic Framework Crystals and Hetero Structures in Confined Microdroplets. J. Am. Chem. Soc. 2013, 135, 14619-14626. [CrossRef] [PubMed]

35. González-Estefan, J.H.; Gonidec, M.; Daro, N.; Marchivie, M.; Chastanet, G. Extreme downsizing in the surfactant-free synthesis of spin-crossover nanoparticles in a microfluidic flow-focusing junction. Chem. Commun. 2018, 54, 8040-8043. [CrossRef] [PubMed]

36. Zheng, B.; Tice, J.D.; Roach, L.S.; Ismagilov, R.F. A droplet-based, composite PDMS/glass capillary microfluidic system for evaluating protein crystallization conditions by microbatch and vapor-diffusion methods with on-chip X-ray diffraction. Angew. Chem. Int. Ed. 2004, 43, 2508-2511. [CrossRef] [PubMed]

37. Laval, P.; Crombez, A.; Salmon, J.-B. Microfluidic Droplet Method for Nucleation Kinetics Measurements. Langmuir 2009, 25, 1836-1841. [CrossRef] [PubMed]

38. Teychené, S.; Biscans, B. Crystal nucleation in a droplet based microfluidic crystallizer. Chem. Eng. Sci. 2012, 77, 242-248. [CrossRef] 
39. Nightingale, A.M.; Bannock, J.H.; Krishnadasan, S.H.; O’Mahony, F.T.F.; Haque, S.A.; Sloan, J.; Drury, C.; McIntyre, R.; DeMello, J.C. Large-scale synthesis of nanocrystals in a multichannel droplet reactor. J. Mater. Chem. A 2013, 12, 4067. [CrossRef]

40. Fu, Y.; Bai, L.; Zhao, S.; Zhang, X.; Jin, Y.; Cheng, Y. Simulation of reactive mixing behaviors inside micro-droplets by a lattice Boltzmann method. Chem. Eng. Sci. 2018, 181, 79-89. [CrossRef]

41. Song, H.; Tice, J.D.; Ismagilov, R.F. A Microfluidic System for Controlling Reaction Networks in Time. Angew. Chem. Int. Ed. 2003, 42, 768-772. [CrossRef]

42. Jiang, L.; Zeng, Y.; Zhou, H.; Qu, J.Y.; Yao, S. Visualizing millisecond chaotic mixing dynamics in microdroplets: A direct comparison of experiment and simulation. Biomicrofluidics 2012, 6, 12810-12812. [CrossRef]

43. Stroock, A.D.; Dertinger, S.K.W.; Ajdari, A.; Mezić, I.; Stone, H.A.; Whitesides, G.M. Chaotic mixer for microchannels. Science 2002, 295, 647-651. [CrossRef] [PubMed]

44. Köhler, J.M.; Abahmane, L.; Wagner, J.; Albert, J.; Mayer, G. Preparation of metal nanoparticles with varied composition for catalytical applications in microreactors. Chem. Eng. Sci. 2008, 63, 5048-5055. [CrossRef]

45. Wagner, J.; Tshikhudo, T.R.; Köhler, J.M. Microfluidic generation of metal nanoparticles by borohydride reduction. Chem. Eng. J. 2008, 135, 104-109. [CrossRef]

46. Kirner, T.; Albert, J.; Günther, M.; Mayer, G.; Reinhäckel, K.; Köhler, J. Static micromixers for modular chip reactor arrangements in two-step reactions and photochemical activated processes. Chem. Eng. J. 2004, 101, 65-74. [CrossRef]

47. Muradoglu, M.; Stone, H.A. Mixing in a drop moving through a serpentine channel: A computational study. Phys. Fluids 2005, 17, 073305. [CrossRef]

48. Song, H.; Bringer, M.R.; Tice, J.D.; Gerdts, C.J.; Ismagilov, R.F. Experimental test of scaling of mixing by chaotic advection in droplets moving through microfluidic channels. Appl. Phys. Lett. 2003, 83, 4664-4666. [CrossRef] [PubMed]

49. Fu, Y.; Wang, H.; Zhang, X.; Bai, L.; Jin, Y.; Cheng, Y. Numerical simulation of liquid mixing inside soft droplets with periodic deformation by a lattice Boltzmann method. J. Taiwan Inst. Chem. Eng. 2018. [CrossRef]

50. Bajer, K.; Moffatt, H.K. On a class of steady confined Stokes flows with chaotic streamlines. J. Fluid Mech. 1990, 212, 337. [CrossRef]

51. Stone, H.A.; Nadim, A.; Strogatz, S.H. Chaotic streamlines inside drops immersed in steady Stokes flows. J. Fluid Mech. 1991, 232, 629. [CrossRef]

52. Stone, Z.B.; Stone, H.A. Imaging and quantifying mixing in a model droplet micromixer. Phys. Fluids 2005, 17, 063103. [CrossRef]

53. Nightingale, A.M.; de Mello, J.C. Segmented Flow Reactors for Nanocrystal Synthesis. Adv. Mater. 2013, 25, 1813-1821. [CrossRef] [PubMed]

54. Hoang, P.H.; Park, H.; Kim, D.-P. Ultrafast and Continuous Synthesis of Unaccommodating Inorganic Nanomaterials in Droplet- and Ionic Liquid-Assisted Microfluidic System. J. Am. Chem. Soc. 2011, 133, 14765-14770. [CrossRef] [PubMed]

55. Paseta, L.; Seoane, B.; Julve, D.; Sebastián, V.; Téllez, C.; Coronas, J. Accelerating the Controlled Synthesis of Metal-Organic Frameworks by a Microfluidic Approach: A Nanoliter Continuous Reactor. ACS Appl. Mater. Interfaces 2013, 5, 9405-9410. [CrossRef] [PubMed]

56. Frenz, L.; Blouwolff, J.; Griffiths, A.D.; Baret, J.C. Microfluidic production of droplet pairs. Langmuir 2008, 24, 12073-12076. [CrossRef] [PubMed]

57. Nightingale, A.M.; Phillips, T.W.; Bannock, J.H.; de Mello, J.C. Controlled multistep synthesis in a three-phase droplet reactor. Nat. Commun. 2014, 5, 3777. [CrossRef] [PubMed]

58. Li, L.; Boedicker, J.Q.; Ismagilov, R.F. Using a Multijunction Microfluidic Device To Inject Substrate into an Array of Preformed Plugs without Cross-Contamination: Comparing Theory and Experiments. Anal. Chem. 2007, 79, 2756-2761. [CrossRef] [PubMed]

(C) 2018 by the authors. Licensee MDPI, Basel, Switzerland. This article is an open access article distributed under the terms and conditions of the Creative Commons Attribution (CC BY) license (http:/ / creativecommons.org/licenses/by/4.0/). 